\title{
Terapia nutricional na reabilitação de doenças crônicas osteoarticulares em idosos
}

\author{
Letícia Mazocco*, Patrícia Chagas**
}

\section{Resumo}

A osteoporose é a doença óssea mais comum em humanos, considerada um dos maiores problemas de saúde pública do mundo, devido a um aumento na expectativa de vida da população. É uma doença que, assim como a artrite reumatoide, a osteoartrite e a gota, tem relação com diversos fatores, entre eles, a nutrição. Este artigo é uma revisão que aborda a importância da dieta na prevenção e no tratamento das doenças crônicas osteoarticulares, em que destacamos as recomendações nutricionais em relação aos macronutrientes, bem como abordamos o importante papel dos alimentos antioxidantes, do cálcio e da Vitamina D, na saúde dos ossos. Uma dieta balanceada e adequada, com ênfase diária em frutas e vegetais frescos, produtos lácteos na forma desnatada, que inclua o azeite de oliva e oleaginosas, poderá auxiliar na manutenção adequada do peso e trazer benefícios antioxidantes e anti-inflamatórios para a prevenção e o tratamento das doenças osteoarticulares.

Palavras-chave: Nutrição. Osteoarticular. Dieta. Osteoporose.

\section{Introdução}

Entre as principais doenças osteoarticulares, podemos destacar osteoporose, artrite reumatoide, osteoartrite, que também é chamada de artrose ou osteoartrose, gota e fibromialgia. Devido à abrangência do tema, vamos focar na nutrição, especificamente em casos de osteoporose, artrite reumatoide, osteoartrite e gota.

A osteoporose é a doença óssea mais comum em humanos e é considerada um dos maiores problemas de saúde pública do mundo, devido a um aumento na expectativa de vida da população (FORTES et al., 2008). Fatores genéticos, contribuem com a densidade mineral óssea (DMO) de $46 \%$ a $62 \%$, e $38 \%$ a $54 \%$ podem ser afetados por fatores relacionados ao estilo de vida, como à nutrição, por exemplo(BARACAT; RADOMINSKI, 2002).

É uma doença que tem relação com diversos fatores, sendo que alguns podem ser modificáveis: hábitos alimenta-

* Nutricionista. Mestranda do Programa de Pós-Graduação em Gerontologia da Universidade Federal de Santa Maria. E-mail: lety.mazocco@gmail.com

** Nutricionista. Professora Adjunta do Departamento de Alimentos e Nutrição e docente do Programa de Pós-Graduação em Gerontologia da Universidade Federal de Santa Maria. E-mail: patriciachagas.ufsm@ hotmail.com

$\rightarrow$ http://dx.doi.org/10.5335/rbceh.v.12i3.6004

Recebido em: 03.11.2015. Aceito em: 03.11.2015. 
res, sedentarismo, composição corporal, tabagismo, corticoterapia prolongada, ingestão excessiva de bebida alcoólica e de café, além da baixa exposição solar (PINHEIRO et al., 2010).

A artrite reumatoide (AR) está associada à osteoporose, constituindo-se em um importante problema de saúde pública. Um pobre estado nutricional tem sido observado em pacientes com $\mathrm{AR}$, que têm um reduzido consumo de carboidratos e de micronutrientes e uma alta ingestão de gordura saturada, contribuindo para o aumento do risco de doenças cardiovasculares (RENNIE et al., 2003). Para o tratamento sintomático da artrite reumatoide, a manipulação dietética está sendo utilizada (MC DOUGALL et al., 2002).

A dieta do mediterrâneo tem sido bastante efetiva na melhora dos sintomas da AR, já que é rica em alimentos de origem vegetal (legumes, frutas, feijão, nozes, sementes e cereais) e peixes, com o azeite de oliva como a principal fonte de gordura, além de um consumo pequeno de carne vermelha e um moderado consumo de vinho como parte das refeições (RAYMAN; CALLAGHAN, 2006).

A forma mais comum de artrite é a osteoartrite (OA), que afeta mais de 20 milhões de indivíduos nos Estados Unidos. Os fatores que contribuem para o desenvolvimento da osteoartrite são: idade avançada, sexo feminino, obesidade, história de trauma, densidade óssea, fraqueza muscular e uso repetitivo das articulações (LI; MICHELETTI, 2011).

A perda de peso corporal tem sido coadjuvante no tratamento da OA, já que com a redução do peso há um alívio nos sintomas e uma melhora das funções (CHRISTENSEN; ASTRUP; BLIDDAL, 2005). Além da perda de peso, a prática de dieta e nutrição adequada tem demonstrado ser um comportamento promissor no alívio moderado dos sintomas da OA e da gota, que é uma artrite inflamatória e está associada à hiperuricemia, causando precipitação de cristais de urato monossódico nas articulações e nos tecidos moles. Estão sendo relacionadas aos componentes da síndrome metabólica: hipertensão, obesidade, diabetes mellitus, hiperlipidemia e também a ingestão de alimentos e bebidas ricos em purina (LI; MICHELETTI, 2011).

As mudanças no estilo de vida dos portadores podem ter o benefício adicional de uma sensação de controle sobre a sua doença, por meio do tratamento por meios não farmacológicos (LI; MICHELETTI, 2011).

\section{Proteínas}

As proteínas (PTNs) são consideradas um elemento fundamental em todos os processos fisiológicos e bioquímicos do organismo, em todas as fases da vida. Exercem função estrutural, enzimática, hormonal, de transporte, de imunidade e contrátil (FRANCISCATO-CAZZOLINO; COMINETTI, 2013). Em relação à contribuição total das proteínas na ingestão calórica, recomenda-se cerca de $10 \%$ a $15 \%$, correspondendo de 0,8 a 0,91 gramas por quilo de peso ao dia (INSTITUT OF MEDICINE, 2005). As principais fontes alimentares de proteínas de origem animal são carnes, ovos, leite e derivados, e de origem vegetal 
são as leguminosas e os cereais integrais (INSTITUT OF MEDICINE, 2005).

A dieta rica em proteína tem aumentado o risco de gota. Para pacientes com a doença, tem sido recomendada uma dieta restrita de purinas, pois estudos metabólicos mostraram que um aumento na carga de purinas purificadas tende a elevar a concentração de ácido úrico (LI; MICHELETTI, 2011).

As PTNs em excesso, principalmente as de origem animal, podem ser prejudiciais à saúde óssea, podendo induzir à acidose metabólica crônica, que, por sua vez, seria responsável pelo aumento da calciúria e pela dissolução mineral acelerada. Em contrapartida, muitos dados experimentais e clínicos publicados indicam que a baixa ingestão de proteína afeta negativamente a saúde óssea (BONJOUR, 2005), pois tem relação com o crescimento e a manutenção dos ossos, atuando como fator protetor da osteoporose (ILICH; KERSTETTER, 2000). Então, dietas hiperproteicas podem ser prejudiciais à saúde óssea (BONJOUR, 2005), assim como uma dieta restrita em PTNs (KERSTETTER; ALLEN, 1994). Acreditamos que uma dieta normoproteica seja a mais adequada nas doenças crônicas osteoarticulares.

\section{Carboidratos}

Os carboidratos são os principais substratos para produzir energia. Exercem inúmeras funções metabólicas (podem afetar a saciedade, a glicemia, a insulinemia e o metabolismo lipídico) e auxiliam no funcionamento do trânsito intestinal. Também podem exercer ação imunorregulatória e influenciar na absorção de cálcio no intestino. $\mathrm{O}$ principal tipo de carboidrato encontrado nos alimentos é o amido (principais fontes: arroz, inhame, mandioca, milho, trigo e batata), seguido dos dissacarídeos, da sacarose (exemplo: cana-de-açúcar) e da lactose (principal fonte: leite) (FRANCISCATO-CAZZOLINO; COMINETTI, 2013).

Em relação ao valor calórico total da dieta, sugere-se que $45 \%$ a $65 \%$ devem ser provenientes de carboidratos (INSTITUT OF MEDICINE, 2005). Dietas com restrição calórica, em que deve-se evitar ingerir carboidratos $(\mathrm{CHO})$ refinados e gordura saturada, demonstraram redução do ácido úrico e da recorrência da gota. Em um grupo de treze homens em dieta, observou-se que os níveis de ácido úrico diminuíram, em média, 1,7 $\mathrm{mg} / \mathrm{dL}$, e o número de ataques de gota mensais caiu de 2,1 para 0,6 , além da perda de peso e da melhora do perfil lipídico (DESSEIN et al., 2000).

Nos últimos trinta anos, houve um aumento drástico no consumo de bebidas adoçadas com açúcar, o que justifica o aumento na prevalência de obesos em todo o mundo. Diversos estudos têm mostrado uma associação significativa entre a ingestão de bebidas açucaradas e doenças cardiometabólicas e obesidade (QI et al., 2012), que é um fator de risco para o desenvolvimento da osteoartrite nos joelhos, mãos e quadril e para a progressão nos joelhos e quadril(DAVIS et al., 1990).

A energia consumida por meio de líquidos pode induzir a uma menor saciedade do que os alimentos sólidos, 
devido à rápida passagem dos líquidos pelo estômago e pelo intestino, podendo levar à redução dos estímulos da saciedade (DIMEGLIO; MATTES, 2000).

\section{Lipídios}

Os lipídios desempenham inúmeras funções no organismo: podem fornecer energia armazenada na forma de trialgliceróis, são elementos de construção importantes das membranas celulares, além de apresentarem importante papel na qualidade dos alimentos, por contribuir na textura, no sabor, em aspectos nutricionais e na densidade calórica. Podem ser classificados em ácidos graxos saturados, poli-insaturados e monoinsaturados (FRANCISCATO-CAZZOLINO; COMINETTI, 2013).

Ácidos graxos saturados (SFA) podem ser divididos em dois grupos: os com cadeia média e os com cadeia longa. Os ácidos graxos de cadeia média não são responsáveis pelo aumento do colesterol plasmático, diferentes dos de cadeia longa, que exercem uma grande influência no colesterol, sendo o ácido graxo palmítico (presente nas carnes) e o mirístico (presente no leite) os que mais contribuem para esse aumento. Consumir esses ácidos graxos até, no máximo, $7 \%$ da dieta, poderá auxiliar na redução do LDL-c, bem como substitui-los por poli-insaturados poderá reduzir o risco cardiovascular (SANTOS et al., 2013).

Ácidos graxos poli-insaturados (Pufa) - os principais são os ácidos graxos Ômega-3 e Ômega-6. Em especial, os ácidos graxos Ômega-3 exercem importante efeito anti-inflamatório, tendo as suas principais fontes alimentares na linhaça e nos peixes (atum, sardinha, cavala, arenque, salmão). Seu consumo é importante na osteoartrite e sua recomendação diária fica em torno de 1-2\% do valor energético total da dieta. Já o ácido graxo Ômega-6, em especial o ácido araquidônico (AA), é substrato para a produção de uma ampla variedade de eicosanoides, e alguns têm características pró-inflamatórias, vasoconstritoras e/ou pró-agregantes. No entanto, o AA também serve de substrato para outros eicosanoides com características anti-inflamatórias/antiagregantes. Assim, seu consumo deve ficar em torno de $5-10 \%$ do valor energético total da dieta (SANTOS et al., 2013).

\section{Ácidos graxos monoinsaturados} (Mufa) - têm uma dupla ligação na cadeia carbônica, sendo o ácido oleico, com maior concentração no azeite de oliva e nas oleaginosas, a melhor fonte. Estudos demonstram que dietas contendo Mufa, em substituição ao Pufa, tornam o LDL menos suscetível à oxidação. Pacientes com doenças osteoarticulares poderão se beneficiar substituindo os SFA por Mufa, pois essa troca poderá colaborar na redução dos níveis pressóricos, na melhora da função endotelial, na redução dos marcadores inflamatórios e de agregação plaquetária. O consumo dietético desses ácidos graxos pode ficar em até $20 \%$ do valor energético total da dieta (SANTOS et al., 2013). 


\section{Antioxidantes}

A vitamina $\mathrm{C}$ é uma vitamina antioxidante solúvel em água, encontrada em frutas cítricas, morango, brócolis, salsa, repolho e pimentões, cuja ingestão moderada (120-200 mg/dia) esteve associada com uma tripla redução no risco de progressão da osteoartrite quando comparada com uma baixa ingestão(MC ALINDON et al., 1996).

Em pacientes com artrite reumatoide, adequadas concentrações teciduais de antioxidantes, podem proporcionar uma importante defesa contra $o$ aumento do estresse oxidativo (RENNIE et al., 2003).

A deficiência de vitamina $\mathrm{E}$ ou a baixa concentração dela nos tecidos, eleva os componentes da resposta inflamatória e suprime os componentes da resposta imunitária em pacientes com artrite reumatoide (RENNIE et al., 2003).

Em um estudo de coorte e em um caso-controle, foi encontrada associação entre o alto consumo de vegetais e a redução do risco de desenvolver a artrite reumatoide (LA VECCHIA; DECARLI; PAGANO, 2008; LINOS et al., 1999). Os pacientes com $A R$, devem satisfazer a ingestão recomendada de antioxidantes por meio da alimentação, já que a suplementação não é recomendada (RENNIE et al., 2003).

\section{Frutas e verduras}

Uma dieta vegetariana rica em vegetais e frutas e pobre em gordura saturada pode reduzir a inflamação corporal total pela alteração dos níveis de ácido araquidônico, ácidos graxos essenciais e antioxidantes, diminuindo também os antígenos alimentares, além de colaborar na manutenção de um peso saudável (SMEDSLUND et al., 2010; ADAM, et al., 2003), sendo um complemento essencial ao tratamento tradicional da artrite reumatoide.

Frutas e legumes apresentaram efeito protetor na osteoartrite de quadril em um estudo de coorte (WILLIAMS; SKINNER; SPECTOR, 2010). Para os pacientes com doenças osteoarticulares, sugerimos o consumo de três porções ao dia de frutas e de três a cinco porções ao dia de vegetais.

\section{Cálcio e vitamina D}

Para os ossos, o nutriente mais importante é o cálcio. Sua ingestão está associada à obtenção do pico da massa óssea, que é obtido entre a adolescência e os 35 anos de idade, além de atuar na prevenção e no tratamento da osteoporose, que tem etiologia multifatorial. $\mathrm{O}$ papel atribuído à nutrição relaciona-se com o desenvolvimento de uma melhor massa óssea possível durante o crescimento e com a proteção do esqueleto contra a perda de cálcio com o passar dos anos (NETO et al., 2002).

A necessidade diária de cálcio para adultos é em torno de $1.000 \mathrm{mg} /$ dia. Quando a absorção intestinal do cálcio encontra-se diminuída ou a taxa de reabsorção óssea está aumentada, como na pós-menopausa, a necessidade aumenta para 1.200 a $1.300 \mathrm{mg} /$ dia (PEREIRA; GENARO; PINHEIRO, 2009).

As principais fontes alimentares de cálcio são o leite e seus derivados e as verduras verde-escuras, como brócolis 
e couve, porém, a biodisponibilidade e a quantidade do cálcio nesses alimentos são menores (PEREIRA; GENARO; PINHEIRO, 2009).

Muitos estudos têm demonstrado que a ingestão de cálcio previne doenças como osteoporose, obesidade, câncer de cólon e hipertensão arterial (HEANEY, 2006), porém, a população tem consumido uma quantidade abaixo do recomendado diariamente, devido à limitada ingestão de leite e derivados, frutas e vegetais, e ao aumento no consumo de refrigerantes, que têm baixo teor de cálcio (NATIONAL INSTITUTE OF HEALTH, 2000).

Em pacientes com AR, a densidade mineral óssea encontrada para a idade foi de $5 \%$ a $15 \%$ menor (ADACHI et al., 1997). A absorção de cálcio pelo intestino é prejudicada pelos corticosteroides, que são utilizados no tratamento da AR (REID; VEALE; FRANCE, 1994).

$A$ vitamina $D$ ativa e modula a síntese do paratormônio (PTH), aumenta a absorção de cálcio pelo intestino, desempenhando um papel fundamental em funções relacionadas ao metabolismo ósseo, estando associada a uma melhor massa óssea e à função muscular, podendo haver relação com a fisiopatogênese de diversas doenças, como a osteopenia e a osteoporose (MAEDA et al., 2014).

A vitamina $D$ pode ser adquirida por meio de alguns alimentos, como o óleo de fígado de bacalhau e peixes gordurosos (salmão, atum, cavala), gema de ovo e cogumelos frescos. Como são poucos os alimentos que têm vitamina $\mathrm{D}$, os seres humanos dependem principalmente da produção cutânea catalisada pelos raios solares UVB (MAEDA et al., 2014).

Condições que limitem a exposição solar podem causar a hipovitaminose $\mathrm{D}$ (REICHRATH; NURNBERG, 2009), que é um problema de saúde pública em todo o mundo, já que é altamente prevalente, assim como no Brasil, podendo acometer mais de $90 \%$ dos indivíduos, dependendo da população estudada (MITHAL et al., 2009).

A complementação das necessidades diárias, assim como o tratamento da deficiência, deve ser realizada para indivíduos com risco para hipovitaminose $\mathrm{D}$ (pacientes com quadro de raquitismo ou osteomalácia, portadores de osteoporose, idosos com história de quedas e fraturas, obesos, grávidas e lactentes, pacientes com síndromes de má-absorção ou que façam uso de medicações que interfiram no metabolismo da vitamina $\mathrm{D}$ (HOLICK et al., 2011), e naqueles com contraindicação clínica para exposição solar, como no câncer de pele, em transplantados ou no lúpus eritematoso sistêmico(MAEDA et al., 2014).

Em pacientes que eram submetidos à terapia prolongada com altas doses de corticoides, devido à variedade de doenças inflamatórias, foram feitos ensaios de suplementação de cálcio e vitamina $\mathrm{D}$ em longo prazo, porém sem efeitos benéficos para a $\mathrm{DMO}$ (ADACHI; IOANNIDIS, 1999).

\section{Conclusão}

Uma dieta balanceada e adequada, com ênfase diária em frutas e vegetais frescos, produtos lácteos na forma des- 
natada, que inclua azeite de oliva e oleaginosas, poderá auxiliar na manutenção adequada do peso e trazer benefícios antioxidantes e anti-inflamatórios para a prevenção e o tratamento das doenças osteoarticulares.

\section{Nutrition therapy in rehabilitation of osteoarticular chronic diseases in elderly}

\section{Abstract}

Osteoporosis is the most common bone disease in human and is beings considered one of the greatest public health problems in the world, due to an increase in life expectancy of the population. It is a disease that, like rheumatoid arthritis, osteoarthritis and gout, is related to several factors, among them, with nutrition. This article is a literature review about the importance of diet in the prevention and treatment of osteoarticular chronic diseases, in which we highlight the nutritional recommendations regarding macronutrients and approaches the important role of antioxidant foods, calcium and Vitamin D in bone`s health. A balanced and proper diet with daily emphasis on fresh fruits and vegetables, dairy products in skimmed form, including olive oil and oilseeds, may assist in proper weight maintenance and bring antioxidant and anti-inflammatory benefits for the prevention and treatment in osteoarticular diseases.

Keywords: Nutrition. Osteoarticular. Diet. Osteoporosis.

\section{Referências}

ADACHI, J. D. et al. Flouride therapy in prevention of rheumatoid arthritis induced bone loss. The Journal of Rheumatology, Toronto, v. 24, p. 2308-2313, 1997.

ADACHI, J. D.; IOANNIDIS, G. Calcium and vitamin $\mathrm{D}$ therapy in corticosteroid-induced bone loss: what is the evidence? Calcified Tissue International, New York, v. 65, p. 332-336, 1999 .

ADAM, O. et al. Anti-inflammatory effects of a low arachidonic acid diet and fish oil in patients with rheumatoid arthritis. Rheumatology International, Berlin, v. 23, n. 1, p. 27-36, 2003.

BARACAT, E.; RADOMINSKI, S. (Coord.). Consenso brasileiro de osteoporose 2002. Revista Brasileira de Reumatologia, São Paulo, v. 42, n. 6, nov./dez, 2002.

BONJOUR, J. P. Dietary protein: an essential nutrient for bone health. Journal of the American College of Nutrition, New York, v. 24, n. 6, p. 526S-36S, 2005.

CHRISTENSEN, R.; ASTRUP, A.; BLIDDAL, H. Weight loss: the treatment of choice for knee osteoarthritis? A randomized trial. Osteoarthritis and Cartilage, London, v. 13, n. 1, p. 20-27, 2005.

DAVIS, M. A. et al. Body fat distribution and osteoarthritis. American Journal of Epidemiology, Baltimore, v. 132, p. 701-707, 1990.

DESSEIN, P. H. et al. Beneficial effects of weight loss associated with moderate calorie/ carbohydrate restriction, and increased proportional intake of protein and unsaturated fat on serum urate and lipoprotein levels in gout: a pilot study. Annals of the Rheumatic Diseases, London, n. 59, p. 539-543, 2000.

DIMEGLIO, D. P.; MATTES, R. D. Liquid versus solid carbohydrate: effects on food intake and body weight. International Journal of Obesity and Related Metabolic Disorders, London, n. 24, p. 794-800, 2000. 
FRANCISCATO-CAZZOLINO, S. M.; COMINETTI, C. Bases bioquímicas e fisiológicas da nutrição: nas diferentes fases da vida, na saúde e na doença. Barueri, SP: Manole, 2013.

FORTES, E. M. et al. Elevada morbimortalidade e reduzida taxa de diagnóstico de osteoporose em idosos com fratura de fêmur proximal na cidade de São Paulo. Arquivos Brasileiros de Endocrinologia e Metabologia, São Paulo, v. 52, n. 7, p. 1106-1114, 2008.

HEANEY, R. P. Calcium intake and disease prevention. Arquivos Brasileiros de Endocrinologia e Metabologia, São Paulo, v. 50, p. 685-693, 2006.

HOLICK, M. F. et al. Endocrine Society. Evaluation, treatment, and prevention of vitamin D deficiency: an Endocrine Society clinical practice guideline. The Journal of Clinical Endocrinology and Metabolism, Springhfield, v. 96, n. 7, p. 1911-1930, 2011.

ILICH, J. Z.; KERSTETTER, J. E. Nutrition in bone health revisited: a story beyond calcium. Journal of the American College of Nutrition, New York, v. 9, n. 6, p. 715-737, 2000 .

INSTITUT OF MEDICINE. Dietary Reference Intakes Tables and Application. 2005. Disponível em: <http://www.iom.edu/Activities/Nutrition/SummaryDRIs/DRITables. aspx>. Acesso em: 25 set. 2014.

KERSTETTER, J. E.; ALLEN, L. H. Protein intake and calcium homeostasis. Advances in Nutritional Research, New York, v. 9, p. 167-181, 1994.

LA VECCHIA, C.; DECARLI, A.; PAGANO, $\mathrm{R}$. Vegetable consumption and risk of chronic disease. Epidemiology, Cambridge, v. 9, p. 208-210, 2008.

LI, S.; MICHELETTI, R. Role of Diet in Rheumatic Disease. Rheumatic Diseases Clinics of North America, Philadelphia, v. 37, p. 119-133, 2011.
LINOS, A. et al. Dietary factors in relation to rheumatoid arthritis: a role for olive oil and cooked vegetables? The American Journal of Clinical Nutrition, Bethesda, v. 70, p. 10771082, 1999.

MAEDA, S. S. et al. Recommendations of the Brazilian Society of Endocrinology and Metabology (SBEM) for the diagnosis and treatment of hypovitaminosis D. Arquivos Brasileiros de Endocrinologia e Metabologia, São Paulo, v. 58, n. 5, p. 411-433, 2014.

MC ALINDON, T. E. et al. Do antioxidant micronutrients protect against the development and progression of knee osteoarthritis? Arthritis and Rheumatism, Atlanta, v. 39, n. 4, p. 648-656, 1996.

MC DOUGALL, J. et al. Effects of a very low - fat, vegan diet in subjects with rheumatoid arthritis. Journal of Alternative and Complementary Medicine, New York, v. 8, n. 1, p. 71-75, 2002.

MITHAL, A. et al. IOF Committee of Scientific Advisors (CSA) Nutrition Working Group. Global vitamin D status and determinants of hypovitaminosis D. Osteoporos International, London, v. 20, n. 11, p. 1807-1820, 2009.

NATIONAL INSTITUTE OF HEALTH. Osteoporosis prevention, diagnosis, and therapy. NIH Consensus Statement, Kensington, v. 17, n. 1, p. 27-29, 2000.

NETO, A. M. P. et al. Brazilian Consensus on Osteoporosis 2002. Revista Brasileira de Reumatologia, São Paulo, v. 42, n. 6, p. 343-54, 2002.

PEREIRA, G. A. P.; GENARO, O. S.; PINHEIRO, M. M. Cálcio dietético - estratégias para otimizar o consumo. Revista Brasileira de Reumatologia, São Paulo, v. 49, n. 2, p. 164-180, 2009.

PINHEIRO, M. M. et al. O impacto da osteoporose no Brasil: dados regionais das fraturas em homens e mulheres adultos - The Brazilian Osteoporosis Study (BRAZOS). Revista Brasileira de Reumatologia, São Paulo, v. 50, n. 2, p. 113-120, 2010. 
QI, Q. et al. Sugar-sweetened beverages and genetic risk of obesity. The New England Journal of Medicine, Boston, v. 15, p. 13871396, 2012.

RAYMAN, M. P.; CALLAGHAN, A. Nutrition and arthritis. Oxford: Blackwell Publishing, 2006.

REICHRATH, J.; NURNBERG, B. Cutaneous vitamin $\mathrm{D}$ synthesis versus skin cancer development: The Janus faces of solar UVradiation. Dermato-Endocrinology, Austin, v. 1, n. 5, p. 253-261, 2009.

REID, I. R.; VEALE, A. G.; FRANCE, J. T. Glucocorticoid osteoporosis. The Journal of Asthma, London, v. 31, p. 7-18, 1994.

RENNIE, K. L. et al. Nutritional management of rheumatoid arthritis: a review of the evidence. Journal of Human Nutrition and Dietetics, Oxford, v. 16, p. 97-109, 2003.

SANTOS, R. D et al. Sociedade Brasileira de Cardiologia. I Diretriz sobre o consume de Gorduras e saúde Cardiovascular. Arquivos Brasileiros de Cardiologia, Rio de Janeiro, v. 100, n. 1, p. 1-40, 2013.

SMEDSLUND, G. et al. Effectiveness and safety of dietary interventions for rheumatoid arthritis: a systematic review of randomized controlled trials. Journal of the American Dietetic Association, Chicago, v. 110, n. 5, p. 727-735, 2010.

WILLIAMS, F. M. K.; SKINNER, J.; SPECTOR, T. D. Dietary garlic and hip osteoarthritis: evidence of a protective effect and putative mechanism of action. BMC Musculoskeletal Disorders, London, v. 11, n. 1, p. 280, 2010. 